\title{
Superposition Coding Based Wireless Network Coding Scheme for Two-Way Cooperative Relaying
}

Megumi KANEKO $^{\dagger \text { a) }}$, Kazunori HAYASHI ${ }^{\dagger}$, Members, and Hideaki SAKAI ${ }^{\dagger}$, Fellow

\begin{abstract}
SUMMARY Recent advances in cooperative communication and wireless Network Coding (NC) may lead to huge performance gains in relay systems. In this context, we focus on the two-way relay scenario, where two nodes exchange information via a common relay. We design a practical Superposition Coding (SC) based NC scheme for Decode-and-Forward (DF) half-duplex relaying, where the goal is to increase the achievable rate. By taking advantage of the direct link and by providing a suboptimal yet efficient power division among the superposed layers, our proposed SC two-way relaying scheme outperforms the reference schemes, including the well-known 3-step DF-NC scheme and the capacity of 2-step schemes for a large set of SNRs, while approaching closely the performance bound. key words: relay system, wireless network coding, superposition coding, cooperative diversity
\end{abstract}

\section{Introduction}

Increasing the data rates and the communication range is a major goal in future wireless communication systems. To attain this goal, the use of relays has gained much interest, due to the low cost and easy deployment of such devices [1]. Many works such as [2]-[4] have shown the efficiency of cooperative diversity, where the broadcast nature of the wireless medium is taken advantage of, as a signal sent by a source node is received both at the relay and at the destination node. By combining the different signals at the destination, significant gains are obtained in terms of throughput/diversity/outage probability. Recently, [5] introduced a wireless relaying scheme based on Superposition Coding (SC), for increasing spectral efficiency. SC was first introduced in the broadcast scenario, where several nodes are served simultaneously by one source [6], [7]. For two destination nodes, the source creates two messages, termed basic and superposed as in [5], that are superposed in the modulation domain and broadcasted. Using successive decoding, the node with a better channel can decode both messages, while the node with a worse channel can only decode the basic message by seeing the superposed one as interference. The proposed scheme in [5] outperforms the conventional multi-hop scheme while performing very closely to the optimal one. Implementation issues of SC are studied in [8].

Another scenario of interest is the two-way relaying, where two nodes exchange information via a common re-

\footnotetext{
Manuscript received May 1, 2010.

Manuscript revised July 16, 2010.

${ }^{\dagger}$ The authors are with the Graduate School of Informatics, Kyoto University, Kyoto-shi, 606-8501 Japan.

a) E-mail: meg@ sys.i.kyoto-u.ac.jp

DOI: 10.1587/transcom.E93.B.3354
}

lay. Huge throughput gains can be obtained by the use of wireless Network Coding (NC) [9]-[14]. Although the concept of NC was originally proposed in [15] for wired communication, it has been the subject of a large number of works in the wireless context (see [16]). Basically, the two nodes first send their messages to the relay, simultaneously as in Amplify-and-Forward (AF) NC or sequentially as in Decode-and-Forward (DF) NC [10], and the relay retransmits a combined version of the two messages into one packet. Then, each node can retrieve the message sent by the other node, by using the prior knowledge of its own message. However, in most previous works that assume DF half-duplex relaying, the two nodes that wish to communicate are assumed to be outside their communication range, whereas in reality, some gain may be achieved by taking advantage of cooperative diversity, e.g., by exploiting the direct link between the two nodes. Note that with AF halfduplex relaying, where the two nodes transmit simultaneously to the relay, the direct link cannot be exploited as neither nodes can transmit and receive at the same time on the same frequency. Some works have considered the use of $\mathrm{NC}$ in other types of cooperative diversity systems, such as user cooperation in the multiple access scenario where several nodes cooperate to deliver their packets to a common destination [17], [18], or in a multi-relay system [12], [19]. In [20], capacity regions were investigated for 3-step and 2-step protocols for the half-duplex two-way relay channel, referred as Time Division Broadcast (TDBC) and Multiple Access Broadcast (MABC), respectively. Due to its 3-step nature, $T D B C$ can take into account any side information that a node may acquire when not transmitting. However, [20] does not specify any practical procedure for attaining these bounds, instead, ideal assumptions are required such as perfect channel knowledge at all nodes, use of random binning and random codes.

In this work, we focus on a two-way relay system between two nodes aided by one relay based on DF halfduplex transmission. The aim is not to determine the capacity of such a system as in [20]. Instead, we design a practical scheme that enhances the achievable rate compared to known schemes, under the fairness constraint that both nodes use the same number of symbol times, as guaranteeing fairness is essential in multi-user systems. We propose a SC based NC scheme that exploits cooperative diversity by taking advantage of the direct link between the two nodes. While a direct extension to the two-way relaying scenario of the SC-relaying scheme in [5] leads to a 4-step scheme, the 
key point of our proposal is that we design an explicit SCbased scheme where we relay the XORed packet of the two superposed messages only, resulting into a 3 step scheme that minimizes the time required for the 3rd step, thereby increasing the overall rate. Note that in order to exploit the direct link, under the assumption of a half-duplex relay, the minimum number of steps is 3 . We refer to our proposed scheme as the SC two-way $(S C-2 W)$ relaying scheme. By efficiently allocating the power among the superposed layers, our scheme outperforms the existing ones in terms of achievable rate, including the well-known conventional DF$\mathrm{NC}$ scheme in 3 steps [10]. By comparison with the reference bounds in [20], we show that our proposed scheme tightly approaches the 3-step bound, and even achieves a higher sum rate than the 2-step bound over a large range of SNRs.

The paper is organized as follows. The system model is introduced in Sect. 2. The proposed scheme is described in Sect. 3 and analyzed in terms of achievable rate in Sect. 4. Reference schemes are defined in Sects. 5, 6, and numerical results are discussed in Sect. 7. Finally, conclusions are drawn and directions for future work are given.

\section{System Model}

As shown in Fig. 1, we consider a system of three nodes $A_{1}$, $A_{2}, A_{\mathrm{R}}$ where $A_{1}$ and $A_{2}$ are the source/destination of the bidirectional traffic, and $A_{\mathrm{R}}$ serves as a relay. If $A_{1}$ transmits a vector $\mathbf{x}_{1}$ of $M$ complex baseband symbols denoted $x_{1}[\mathrm{~m}]$, $m \in[1, M]$, the received signal at $A_{\mathrm{R}}$ and $A_{2}$ is given by

$$
\mathbf{y}_{1 \mathrm{R}}=h_{1} \mathbf{x}_{1}+\mathbf{z}_{\mathrm{R}}^{(1)}, \quad \mathbf{y}_{12}=h_{0} \mathbf{x}_{1}+\mathbf{z}_{2}^{(1)},
$$

and if $A_{\mathrm{R}}$ transmits a vector $\mathbf{x}_{\mathrm{R}}$ of $M_{\mathrm{R}}$ complex baseband symbols denoted $x_{\mathrm{R}}[m], m \in\left[1, M_{\mathrm{R}}\right]$, the received signal at $A_{1}$ and $A_{2}$ is given by

$$
\mathbf{y}_{\mathrm{R} 1}=h_{1} \mathbf{x}_{\mathrm{R}}+\mathbf{z}_{1}^{(2)}, \quad \mathbf{y}_{\mathrm{R} 2}=h_{2} \mathbf{x}_{\mathrm{R}}+\mathbf{z}_{2}^{(2)} .
$$

Similar equations are obtained for the transmissions originated from $A_{2}$. Here, $h_{i}$ denotes the complex channel coefficient for $i \in\{0,1,2\}$, where we assume channel reciprocity. Vector $\mathbf{z}_{\mathbf{I}}, I \in\{1,2, R\}$, is the complex additive white Gaussian noise with a circular-symmetric distribution $C \mathcal{N}\left(\mathbf{0}, \sigma^{2} \mathbf{I}\right)$. The transmitted symbols have a mean zero, e.g., $\mathrm{E}\left\{x_{\mathrm{I}}[m]\right\}=0$ and a power normalized to one, e.g., $\mathrm{E}\left\{\left|x_{\mathrm{I}}[m]\right|^{2}\right\}=1$. Thus, the link SNRs are given by $\gamma_{i}=\frac{\left|h_{i}\right|^{2}}{\sigma^{2}}$, $i \in\{0,1,2\}$. The capacity of each link is given by

$$
C\left(\gamma_{i}\right)=\log _{2}\left(1+\gamma_{i}\right) \quad[\mathrm{bits} / \mathrm{s}],
$$

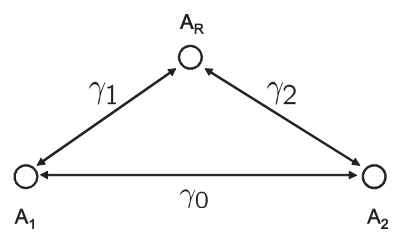

Fig. 1 System model. for a normalized bandwidth of $1 \mathrm{~Hz}$. In this work, we assume that the relayed links are better than the direct link,

$$
\gamma_{1}>\gamma_{0}, \quad \gamma_{2}>\gamma_{0},
$$

which justifies the use of a relay. Moreover, we assume

$$
\gamma_{2} \geq \gamma_{1},
$$

without loss of generality. The SNRs are assumed to remain constant during the steps explained next. Moreover, it is assumed that each node knows all received SNRs perfectly.

\section{Description of the Proposed Scheme}

As shown in Fig. 2, the proposed scheme is composed of the following three steps:

Step 1: Node $A_{1}$ sends $\mathbf{x}_{1}$, composed of the basic message $\mathbf{x}_{\mathrm{b}_{1}}$ and the superposed message $\mathbf{x}_{\mathrm{s}_{1}}$, both intended for $A_{2}$,

$$
\mathbf{x}_{1}=\sqrt{1-\alpha_{1}} \mathbf{x}_{\mathrm{b}_{1}}+\sqrt{\alpha_{1}} \mathbf{x}_{\mathrm{s}_{1}},
$$

where $\alpha_{1} \in[0,1]$ denotes the power allocation ratio between the basic and superposed messages. Then, $A_{\mathrm{R}}$ receives

$$
\mathbf{y}_{1 \mathrm{R}}=h_{1}\left(\sqrt{1-\alpha_{1}} \mathbf{x}_{\mathrm{b}_{1}}+\sqrt{\alpha_{1}} \mathbf{x}_{\mathrm{s}_{1}}\right)+\mathbf{z}_{\mathrm{R}}^{(1)} .
$$

From $\mathbf{y}_{1 \mathrm{R}}, A_{\mathrm{R}}$ first decodes $\mathbf{x}_{\mathrm{b}_{1}}$ and cancels it, obtaining

$$
\hat{\mathbf{y}}_{1 \mathrm{R}}=h_{1} \sqrt{\alpha_{1}} \mathbf{x}_{\mathrm{s}_{1}}+\mathbf{z}_{\mathrm{R}}^{(1)},
$$

from which $\mathbf{x}_{\mathrm{s}_{1}}$ is decoded. On the other hand, $A_{2}$ receives

$$
\mathbf{y}_{12}=h_{0}\left(\sqrt{1-\alpha_{1}} \mathbf{x}_{\mathrm{b}_{1}}+\sqrt{\alpha_{1}} \mathbf{x}_{\mathrm{s}_{1}}\right)+\mathbf{z}_{2}^{(1)}
$$

and keeps it in memory.

Step 2: Similarly, node $A_{2}$ sends $\mathbf{x}_{2}$,

$$
\mathbf{x}_{2}=\sqrt{1-\alpha_{2}} \mathbf{x}_{\mathrm{b}_{2}}+\sqrt{\alpha_{2}} \mathbf{x}_{\mathrm{s}_{2}},
$$

where $\alpha_{2} \in[0,1]$ denotes the power allocation ratio between the basic message $\mathbf{x}_{\mathrm{b}_{2}}$ and the superposed message $\mathbf{x}_{\mathrm{s}_{2}}$, both intended for node $A_{1}$. Then, relay node $A_{\mathrm{R}}$ receives

$$
\mathbf{y}_{2 \mathrm{R}}=h_{2}\left(\sqrt{1-\alpha_{2}} \mathbf{x}_{\mathrm{b}_{2}}+\sqrt{\alpha_{2}} \mathbf{x}_{\mathrm{s}_{2}}\right)+\mathbf{z}_{\mathrm{R}}^{(2)} .
$$

From $\mathbf{y}_{2 \mathrm{R}}, A_{\mathrm{R}}$ first decodes $\mathbf{x}_{\mathrm{b}_{2}}$ and cancels it, and then decodes $\mathbf{x}_{\mathrm{s}_{2}}$. On the other hand, node $A_{1}$ receives

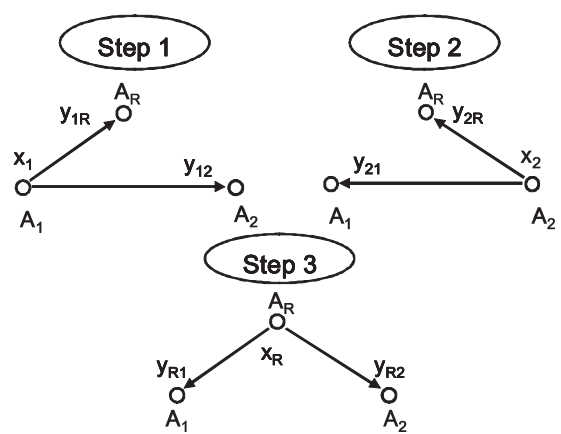

Fig. 2 Steps of the proposed scheme. 


$$
\mathbf{y}_{21}=h_{0}\left(\sqrt{1-\alpha_{2}} \mathbf{x}_{\mathrm{b}_{2}}+\sqrt{\alpha_{2}} \mathbf{x}_{\mathrm{s}_{2}}\right)+\mathbf{z}_{1}^{(2)}
$$

and keeps it in memory.

Step 3: From the messages $\mathbf{x}_{\mathrm{s}_{1}}$ and $\mathbf{x}_{\mathrm{s}_{2}}$ decoded in Steps 1 and $2, A_{\mathrm{R}}$ creates two messages of the same bit length by padding the shorter one with zeros. The packets obtained are denoted $\mathbf{b}_{\mathrm{s}_{1}}$ and $\mathbf{b}_{\mathrm{s}_{2}}$. Then, a new packet $\mathbf{b}_{\mathrm{R}}$ is obtained by XORing these two packets,

$$
\mathbf{b}_{\mathrm{R}}=\mathbf{b}_{\mathrm{s}_{1}} \oplus \mathbf{b}_{\mathrm{s}_{2}} \text {. }
$$

Denoting $\mathbf{x}_{\mathrm{R}}$ the message corresponding to $\mathbf{b}_{\mathrm{R}}, A_{\mathrm{R}}$ sends $\mathbf{x}_{\mathrm{R}}$, received by $A_{1}$ and $A_{2}$. From the received signal at $A_{1}$

$$
\mathbf{y}_{\mathrm{R} 1}=h_{1} \mathbf{x}_{\mathrm{R}}+\mathbf{z}_{1}^{(3)}
$$

$A_{1}$ first decodes $\mathbf{x}_{\mathrm{R}}$ then, XORing packets $\mathbf{b}_{\mathrm{s}_{1}}$ and $\mathbf{b}_{\mathrm{R}}$ corresponding to symbol messages $\mathbf{x}_{\mathrm{s}_{1}}$ and $\mathbf{x}_{\mathrm{R}}, A_{1}$ obtains

$$
\mathbf{b}_{\mathrm{R}} \oplus \mathbf{b}_{\mathrm{s}_{1}}=\left(\mathbf{b}_{\mathrm{s}_{1}} \oplus \mathbf{b}_{\mathrm{s}_{2}}\right) \oplus \mathbf{b}_{\mathrm{s}_{1}}=\mathbf{b}_{\mathrm{s}_{2}} \text {. }
$$

From $\mathbf{b}_{\mathrm{s}_{2}}, A_{1}$ creates $\mathbf{x}_{\mathrm{s}_{2}}$. Then, canceling $\mathbf{x}_{\mathrm{s}_{2}}$ from the signal received in Step 2, $A_{1}$ obtains

$$
\mathbf{y}_{2}=\mathbf{y}_{21}-h_{0} \sqrt{\alpha_{2}} \mathbf{x}_{\mathrm{s}_{2}}=h_{0} \sqrt{1-\alpha_{2}} \mathbf{x}_{\mathrm{b}_{2}}+\mathbf{z}_{1}^{(2)}
$$

from which $\mathbf{x}_{\mathrm{b}_{2}}$ is decoded. Similarly, $A_{2}$ decodes $\mathbf{x}_{\mathrm{R}}$ from

$$
\mathbf{y}_{\mathrm{R} 2}=h_{2} \mathbf{x}_{\mathrm{R}}+\mathbf{z}_{2}^{(3)} \text {, }
$$

then obtains $\mathbf{x}_{\mathrm{s}_{1}}$ from the decoded packet $\mathbf{b}_{\mathrm{s}_{1}}$. Then, canceling $\mathbf{x}_{\mathrm{s}_{1}}$ from the signal received in Step 1, $A_{2}$ obtains

$$
\mathbf{y}_{1}=\mathbf{y}_{12}-h_{0} \sqrt{\alpha_{1}} \mathbf{x}_{\mathrm{s}_{1}}=h_{0} \sqrt{1-\alpha_{1}} \mathbf{x}_{\mathrm{b}_{1}}+\mathbf{z}_{2}^{(1)},
$$

and finally decodes $\mathbf{x}_{\mathrm{b}_{1}}$.

\section{Analysis of the Proposed Scheme}

We derive the expression of the achievable rate of the proposed scheme under the fairness constraint that each node $A_{1}$ and $A_{2}$ transmits over the same number of symbol times $M$. We denote $R_{\mathrm{b} j}$ and $R_{\mathrm{s} j}$ the rates of the basic and superposed messages from node $A_{j}, j=\{1,2\}$, respectively. Then, $M\left(R_{\mathrm{b}_{1}}+R_{\mathrm{s}_{1}}\right)$ bits are sent by $A_{1}$ and $M\left(R_{\mathrm{b}_{2}}+R_{\mathrm{s}_{2}}\right)$ bits are sent by $A_{2}$. In Step 3 , the relay forwards one packet whose length is equal to the longer one among $\mathbf{b}_{\mathrm{s}_{1}}$ and $\mathbf{b}_{\mathrm{s}_{2}}$, thus the amount of bits sent by $A_{\mathrm{R}}$ is equal to $M \max \left(R_{\mathrm{s}_{1}}, R_{\mathrm{S}_{2}}\right)$. Denoting $R_{\mathrm{r}_{j}}$ the rate on link $A_{\mathrm{R}}-A_{j}, j=\{1,2\}$, the rate at which $\mathbf{x}_{\mathrm{R}}$ is sent is adapted to the worse link among $A_{\mathrm{R}}-A_{1}$ and $A_{\mathrm{R}}-A_{2}$, i.e., to $\min \left(R_{\mathrm{r}_{1}}, R_{\mathrm{r}_{2}}\right)$. Thus, the number of symbol times required in Step 3 is $M_{\mathrm{R}}=\frac{M \max \left(R_{\mathrm{s}_{1}}, R_{\mathrm{s}_{2}}\right)}{\min \left(R_{\mathrm{r}_{1}}, R_{\mathrm{r}_{2}}\right)}$. In total, $M\left(R_{\mathrm{b}_{1}}+R_{\mathrm{S}_{1}}+R_{\mathrm{b}_{2}}+R_{\mathrm{S}_{2}}\right)$ bits are sent during $2 M+M_{\mathrm{R}}$ symbol times, giving

$$
R_{\mathrm{SC}-2 \mathrm{~W}}=\frac{R_{\mathrm{b}_{1}}+R_{\mathrm{S}_{1}}+R_{\mathrm{b}_{2}}+R_{\mathrm{s}_{2}}}{2+\frac{\max \left(R_{\mathrm{S}_{1}}, R_{\mathrm{S}_{2}}\right)}{\min \left(R_{\mathrm{r}_{1}}, R_{\mathrm{r}_{2}}\right)}} .
$$

The goal is to determine the optimal power ratios $\alpha_{1}$, $\alpha_{2}$ that maximize $R_{\mathrm{SC}-2 \mathrm{~W}}$. First, from (19) we observe that
$R_{\mathrm{SC}-2 \mathrm{~W}}$ increases by maximizing $\min \left(R_{\mathrm{r}_{1}}, R_{\mathrm{r}_{2}}\right)$, so from assumption (5), $\min \left(R_{\mathrm{r}_{1}}, R_{\mathrm{r}_{2}}\right)=C\left(\gamma_{1}\right)$. Next, we focus on the behavior of $R_{\mathrm{SC}-2 \mathrm{~W}}$ in function of $R_{\mathrm{S}_{1}}, R_{\mathrm{S}_{2}}$ by writing

$$
R_{\mathrm{SC}-2 \mathrm{~W}}=\left(R_{\mathrm{b}_{1}}+R_{\mathrm{b}_{2}}\right) \times \frac{1+\frac{R_{\mathrm{s}_{1}}+R_{\mathrm{s}_{2}}}{R_{\mathrm{b}_{1}}+R_{\mathrm{b}_{2}}}}{2+\frac{\max \left(R_{\mathrm{S}_{1}}, R_{\mathrm{S}_{2}}\right)}{C\left(\gamma_{1}\right)}} .
$$

If $R_{\mathrm{S}_{1}} \geq R_{\mathrm{S}_{2}}, R_{\mathrm{SC}-2 \mathrm{~W}}$ increases with $R_{\mathrm{S}_{2}}$ and we can show that $\frac{\partial R_{\mathrm{SC}-2 \mathrm{~W}}}{\partial R_{\mathrm{S}_{1}}} \geq 0$ if $2 C\left(\gamma_{1}\right) \geq R_{\mathrm{b}_{1}}+R_{\mathrm{b}_{2}}+R_{\mathrm{S}_{2}}$, which is satisfied a fortiori if $2 C\left(\gamma_{1}\right) \geq R_{\mathrm{b}_{1}}+R_{\mathrm{b}_{2}}+R_{\mathrm{s}_{1}}$. On the other hand, if $R_{\mathrm{S}_{2}} \geq R_{\mathrm{S}_{1}}$, $R_{\mathrm{SC}-2 \mathrm{~W}}$ increases with $R_{\mathrm{S}_{1}}$, and we can show that $\frac{\partial R_{\mathrm{SC}-2 \mathrm{~W}}}{\partial R_{\mathrm{S}_{2}}} \geq 0$ if $2 C\left(\gamma_{1}\right) \geq R_{\mathrm{b}_{1}}+R_{\mathrm{b}_{2}}+R_{\mathrm{S}_{1}}$. Thus, in both cases, $R_{\mathrm{SC}-2 \mathrm{~W}}$ is an increasing function of $R_{\mathrm{s}_{1}}$ and $R_{\mathrm{s}_{2}}$ if

$$
2 C\left(\gamma_{1}\right) \geq R_{\mathrm{b}_{1}}+R_{\mathrm{b}_{2}}+R_{\mathrm{s}_{1}} \text {. }
$$

In the proposed scheme, the rates follow the constraints below. For the transmissions from $A_{1}, j=1$ and $k=2$, and

$$
\begin{aligned}
& R_{\mathrm{b}_{j}} \leq C\left(\frac{\left(1-\alpha_{j}\right) \gamma_{j}}{1+\alpha_{j} \gamma_{j}}\right) \stackrel{\text { def }}{=} R_{\mathrm{b}_{j}}^{\mathrm{UA}}\left(\alpha_{j}\right) \\
& R_{\mathrm{s}_{j}} \leq C\left(\alpha_{j} \gamma_{j}\right) \stackrel{\text { def }}{=} R_{\mathrm{s}_{j}}^{\mathrm{U}}\left(\alpha_{j}\right) \\
& R_{\mathrm{b}_{j}} \leq C\left(\left(1-\alpha_{j}\right) \gamma_{0}\right) \stackrel{\text { def }}{=} R_{\mathrm{b}_{j}}^{\mathrm{UB}}\left(\alpha_{j}\right) \\
& R_{\mathrm{r}_{k}} \leq C\left(\gamma_{k}\right) \stackrel{\text { def }}{=} R_{\mathrm{r}_{k}}^{\mathrm{U}},
\end{aligned}
$$

where (22) ensures that $A_{\mathrm{R}}$ can decode $\mathbf{x}_{\mathrm{b}_{j}}$ by seeing $h_{j} \sqrt{\alpha_{j}} \mathbf{x}_{\mathrm{s}_{j}}$ as interference in Eq. (7); likewise, (23) ensures that $A_{\mathrm{R}}$ can decode $\mathbf{x}_{\mathrm{s}_{j}}$, and (24), (25) ensure that $A_{k}$ can decode $\mathbf{x}_{\mathrm{b}_{j}}$ and $\mathbf{x}_{\mathrm{R}}$. For the transmissions from $A_{2}$, we obtain the same set of constraints with $j=2$ and $k=1$.

As $R_{\mathrm{b}_{1}} \leq \min \left(R_{\mathrm{b}_{1}}^{\mathrm{UA}}, R_{\mathrm{b}_{1}}^{\mathrm{UB}}\right)$, then we have $R_{\mathrm{b}_{1}}+R_{\mathrm{s}_{1}} \leq$ $R_{\mathrm{b}_{1}}^{\mathrm{UA}}+R_{\mathrm{s}_{1}}^{\mathrm{U}}=C\left(\gamma_{1}\right)$. Moreover, $R_{\mathrm{b}_{2}} \leq R_{\mathrm{b}_{2}}^{\mathrm{UB}}<C\left(\gamma_{0}\right)$ from (24). Thus, (21) is satisfied as long as $2 C\left(\gamma_{1}\right) \geq C\left(\gamma_{1}\right)+C\left(\gamma_{0}\right)$, e.g., $\gamma_{1} \geq \gamma_{0}$, which is always true given assumption (4). Thus, $R_{\mathrm{SC}-2 \mathrm{~W}}$ is an increasing function of $R_{\mathrm{S}_{1}}, R_{\mathrm{S}_{2}}$ so we set $R_{\mathrm{S}_{1}}=R_{\mathrm{S}_{1}}^{\mathrm{U}}, R_{\mathrm{S}_{2}}=R_{\mathrm{S}_{2}}^{\mathrm{U}}$, and the overall rate can be written

$$
R_{\mathrm{SC}-2 \mathrm{~W}}=\frac{\left(R_{\mathrm{b}_{1}}+C\left(\alpha_{1} \gamma_{1}\right)+R_{\mathrm{b}_{2}}+C\left(\alpha_{2} \gamma_{2}\right)\right) C\left(\gamma_{1}\right)}{2 C\left(\gamma_{1}\right)+\max \left(C\left(\alpha_{1} \gamma_{1}\right), C\left(\alpha_{2} \gamma_{2}\right)\right)} \text {. }
$$

We can see that $R_{\mathrm{SC}-2 \mathrm{~W}}$ increases as $R_{\mathrm{b}_{1}}$ and $R_{\mathrm{b}_{2}}$ increase. However, given the constraints (22), (24), we need to consider the following four cases:

$$
\begin{aligned}
& \text { (a) } R_{\mathrm{b}_{1}}^{\mathrm{UA}}\left(\alpha_{1}\right) \leq R_{\mathrm{b}_{1}}^{\mathrm{UB}}\left(\alpha_{1}\right) \quad \& \quad R_{\mathrm{b}_{2}}^{\mathrm{UA}}\left(\alpha_{2}\right) \leq R_{\mathrm{b}_{2}}^{\mathrm{UB}}\left(\alpha_{2}\right) \\
& \Longleftrightarrow \alpha_{1}^{*} \leq \alpha_{1} \leq 1 \quad \& \quad \alpha_{2}^{*} \leq \alpha_{2} \leq 1 \\
& \text { (b) } R_{\mathrm{b}_{1}}^{\mathrm{UA}}\left(\alpha_{1}\right) \geq R_{\mathrm{b}_{1}}^{\mathrm{UB}}\left(\alpha_{1}\right) \quad \& \quad R_{\mathrm{b}_{2}}^{\mathrm{UA}}\left(\alpha_{2}\right) \leq R_{\mathrm{b}_{2}}^{\mathrm{UB}}\left(\alpha_{2}\right) \\
& \Longleftrightarrow 0 \leq \alpha_{1} \leq \alpha_{1}^{*} \quad \& \quad \alpha_{2}^{*} \leq \alpha_{2} \leq 1 \\
& \text { (c) } R_{\mathrm{b}_{1}}^{\mathrm{UA}}\left(\alpha_{1}\right) \leq R_{\mathrm{b}_{1}}^{\mathrm{UB}}\left(\alpha_{1}\right) \quad \& \quad R_{\mathrm{b}_{2}}^{\mathrm{UA}}\left(\alpha_{2}\right) \geq R_{\mathrm{b}_{2}}^{\mathrm{UB}}\left(\alpha_{2}\right) \\
& \Longleftrightarrow \alpha_{1}^{*} \leq \alpha_{1} \leq 1 \quad \& \quad 0 \leq \alpha_{2} \leq \alpha_{2}^{*} \\
& \text { (d) } R_{\mathrm{b}_{1}}^{\mathrm{UA}}\left(\alpha_{1}\right) \geq R_{\mathrm{b}_{1}}^{\mathrm{UB}}\left(\alpha_{1}\right) \quad \& \quad R_{\mathrm{b}_{2}}^{\mathrm{UA}}\left(\alpha_{2}\right) \geq R_{\mathrm{b}_{2}}^{\mathrm{UB}}\left(\alpha_{2}\right) \text {. } \\
& \Longleftrightarrow 0 \leq \alpha_{1} \leq \alpha_{1}^{*} \quad \& \quad 0 \leq \alpha_{2} \leq \alpha_{2}^{*},
\end{aligned}
$$

where we have defined 


$$
\alpha_{j}^{*}=\frac{1}{\gamma_{0}}-\frac{1}{\gamma_{j}}
$$

As we have the constraint $0 \leq \alpha_{j}^{*} \leq 1$, (31) is valid for SNRs satisfying $\frac{\gamma_{j}}{1+\gamma_{j}} \leq \gamma_{0} \leq \gamma_{j}$, which we assume in the following. Next, we derive the optimal value of $R_{\mathrm{SC}-2 \mathrm{~W}}$ by considering each case.

(a) $\alpha_{1}^{*} \leq \alpha_{1} \leq 1 \& \alpha_{2}^{*} \leq \alpha_{2} \leq 1$ : In this case, we have $R_{\mathrm{b}_{1}}+R_{\mathrm{s}_{1}}=C\left(\frac{\left(1-\alpha_{1}\right) \gamma_{1}}{1+\alpha_{1} \gamma_{1}}\right)+C\left(\alpha_{1} \gamma_{1}\right)=C\left(\gamma_{1}\right)$ and $R_{\mathrm{b}_{2}}+R_{\mathrm{s}_{2}}=$ $C\left(\gamma_{2}\right)$. Thus, the overall rate becomes

$$
R_{\mathrm{SC}-2 \mathrm{~W}}=\frac{\left[C\left(\gamma_{1}\right)+C\left(\gamma_{2}\right)\right] C\left(\gamma_{1}\right)}{2 C\left(\gamma_{1}\right)+\max \left(C\left(\alpha_{1} \gamma_{1}\right), C\left(\alpha_{2} \gamma_{2}\right)\right)},
$$

maximized when $\max \left(C\left(\alpha_{1} \gamma_{1}\right), C\left(\alpha_{2} \gamma_{2}\right)\right)$ is minimal, i.e.,

$$
\alpha_{1}=\alpha_{1}^{*}, \quad \alpha_{2}=\alpha_{2}^{*} .
$$

The assumption $\gamma_{1}<\gamma_{2}$ implies $\alpha_{1}^{*}<\alpha_{2}^{*}$, and so $C\left(\alpha_{1}^{*} \gamma_{1}\right)<$ $C\left(\alpha_{2}^{*} \gamma_{2}\right)$. Finally, we obtain

$$
R_{\mathrm{SC}-2 \mathrm{~W}}^{(a)}=\frac{\left[C\left(\gamma_{1}\right)+C\left(\gamma_{2}\right)\right] C\left(\gamma_{1}\right)}{2 C\left(\gamma_{1}\right)+C\left(\alpha_{2}^{*} \gamma_{2}\right)} .
$$

(b) $0 \leq \alpha_{1} \leq \alpha_{1}^{*} \& \alpha_{2}^{*} \leq \alpha_{2} \leq 1$ : In this case, we have $R_{\mathrm{b}_{1}}+R_{\mathrm{s}_{1}}=C\left(\left(1-\alpha_{1}\right) \gamma_{0}\right)+C\left(\alpha_{1} \gamma_{1}\right)$ and $R_{\mathrm{b}_{2}}+R_{\mathrm{s}_{2}}=C\left(\gamma_{2}\right)$. From $\gamma_{1}<\gamma_{2}$, we have $\alpha_{1}^{*}<\alpha_{2}^{*}$, hence $\alpha_{1}<\alpha_{2}$ in this region, which implies $C\left(\alpha_{1} \gamma_{1}\right)<C\left(\alpha_{2} \gamma_{2}\right)$, giving

$$
R_{\mathrm{SC}-2 \mathrm{~W}}=\frac{\left[C\left(\left(1-\alpha_{1}\right) \gamma_{0}\right)+C\left(\alpha_{1} \gamma_{1}\right)+C\left(\gamma_{2}\right)\right] C\left(\gamma_{1}\right)}{2 C\left(\gamma_{1}\right)+C\left(\alpha_{2} \gamma_{2}\right)} \text {. }
$$

$R_{\mathrm{SC}-2 \mathrm{~W}}$ is a decreasing function of $\alpha_{2}$, so it is optimal at

$$
\alpha_{2}=\alpha_{2}^{*} \text {. }
$$

Let us define $\eta\left(\gamma_{j}\right)=C\left(\left(1-\alpha_{j}\right) \gamma_{0}\right)+C\left(\alpha_{j} \gamma_{j}\right)$, equal to

$$
\begin{aligned}
\eta\left(\gamma_{j}\right)= & \log _{2}\left\{-\gamma_{0} \gamma_{j}\left(\alpha_{j}-\frac{1}{2}\left(1+\frac{1}{\gamma_{0}}-\frac{1}{\gamma_{j}}\right)\right)^{2}\right. \\
& \left.+\frac{\gamma_{0} \gamma_{j}}{4}\left(1+\frac{1}{\gamma_{0}}-\frac{1}{\gamma_{j}}\right)^{2}+1+\gamma_{0}\right\} .
\end{aligned}
$$

The behavior of $R_{\mathrm{SC}-2 \mathrm{~W}}$ in function of $\alpha_{1}$ is equivalent to that of $\eta\left(\gamma_{1}\right)$, maximized when $\alpha_{1}=\tilde{\alpha}_{1}$ where we define

$$
\tilde{\alpha}_{j}=\frac{1}{2}\left(1+\frac{1}{\gamma_{0}}-\frac{1}{\gamma_{j}}\right) \text {. }
$$

However, $0 \leq \alpha_{1}^{*} \leq 1$ implies that $\alpha_{1}^{*} \leq \tilde{\alpha}_{1}$. Given the region of values of $\alpha_{1}$, the optimal value is here

$$
\alpha_{1}=\alpha_{1}^{*},
$$

and the overall rate becomes

$$
R_{\mathrm{SC}-2 \mathrm{~W}}^{(b)}=\frac{\left[C\left(\left(1-\alpha_{1}^{*}\right) \gamma_{0}\right)+C\left(\alpha_{1}^{*} \gamma_{1}\right)+C\left(\gamma_{2}\right)\right] C\left(\gamma_{1}\right)}{2 C\left(\gamma_{1}\right)+C\left(\alpha_{2}^{*} \gamma_{2}\right)}
$$

(c) $\alpha_{1}^{*} \leq \alpha_{1} \leq 1 \& 0 \leq \alpha_{2} \leq \alpha_{2}^{*}$ : In this case, we have $R_{\mathrm{b}_{1}}+$ $R_{\mathrm{s}_{1}}=C\left(\gamma_{1}\right)$ and $R_{\mathrm{b}_{2}}+R_{\mathrm{s}_{2}}=C\left(\left(1-\alpha_{2}\right) \gamma_{0}\right)+C\left(\alpha_{2} \gamma_{2}\right)$. We need to consider subcases concerning $\max \left(C\left(\alpha_{1} \gamma_{1}\right), C\left(\alpha_{2} \gamma_{2}\right)\right)$. (c-1) If $C\left(\alpha_{1} \gamma_{1}\right) \geq C\left(\alpha_{2} \gamma_{2}\right)$ : then

$$
R_{\mathrm{SC}-2 \mathrm{~W}}=\frac{\left[C\left(\gamma_{1}\right)+C\left(\left(1-\alpha_{2}\right) \gamma_{0}\right)+C\left(\alpha_{2} \gamma_{2}\right)\right] C\left(\gamma_{1}\right)}{2 C\left(\gamma_{1}\right)+C\left(\alpha_{1} \gamma_{1}\right)},
$$

which is a decreasing function of $\alpha_{1}$, hence we set

$$
\alpha_{1}=\alpha_{1}^{*} \text {. }
$$

As a function of $\alpha_{2}, R_{\mathrm{SC}-2 \mathrm{~W}}$ follows the behavior of $\eta\left(\gamma_{2}\right)$ which is maximized for $\alpha_{2}=\tilde{\alpha}_{2}$ defined in (35), (36). But we observe that $\alpha_{2}^{*} \leq \tilde{\alpha}_{2}$, so $\tilde{\alpha}_{2}$ is out of range. Moreover, from the conditions $C\left(\alpha_{1} \gamma_{1}\right) \geq C\left(\alpha_{2} \gamma_{2}\right)$ and $\alpha_{1}=\alpha_{1}^{*}$, we observe that $\alpha_{1}^{*} \gamma_{1} \geq \alpha_{2} \gamma_{2} \Leftrightarrow \alpha_{2} \leq \hat{\alpha}_{2}$, where we define

$$
\hat{\alpha}_{2}=\frac{\gamma_{1}-\gamma_{0}}{\gamma_{0} \gamma_{2}} \text {. }
$$

Furthermore, we see that $\hat{\alpha}_{2} \leq \frac{\gamma_{2}-\gamma_{0}}{\gamma_{0} \gamma_{2}}=\alpha_{2}^{*}$ since we assumed $\gamma_{1}<\gamma_{2}$. Thus, the optimal value is here

$$
\alpha_{2}=\hat{\alpha}_{2},
$$

and $R_{\mathrm{SC}-2 \mathrm{~W}}$ becomes

$$
R_{\mathrm{SC}-2 \mathrm{~W}}^{(c 1)}=\frac{\left[C\left(\gamma_{1}\right)+C\left(\left(1-\hat{\alpha}_{2}\right) \gamma_{0}\right)+C\left(\hat{\alpha}_{2} \gamma_{2}\right)\right] C\left(\gamma_{1}\right)}{2 C\left(\gamma_{1}\right)+C\left(\alpha_{1}^{*} \gamma_{1}\right)}
$$

(c-2) If $C\left(\alpha_{1} \gamma_{1}\right)<C\left(\alpha_{2} \gamma_{2}\right)$, then $R_{\mathrm{SC}-2 \mathrm{~W}}$ becomes

$$
R_{\mathrm{SC}-2 \mathrm{~W}}=\frac{\left[C\left(\gamma_{1}\right)+C\left(\left(1-\alpha_{2}\right) \gamma_{0}\right)+C\left(\alpha_{2} \gamma_{2}\right)\right] C\left(\gamma_{1}\right)}{2 C\left(\gamma_{1}\right)+C\left(\alpha_{2} \gamma_{2}\right)},
$$

which only depends on $\alpha_{2}$. To study the behavior of $R_{\mathrm{SC}-2 \mathrm{~W}}$ in function of $\alpha_{2}$, we determine its partial derivative. It can be shown to follow the behavior of $f\left(\alpha_{2}\right)$, defined as $f\left(\alpha_{2}\right)=$ $f_{2}\left(\alpha_{2}\right)+f_{1}\left(\alpha_{2}\right)+f_{0}\left(\alpha_{2}\right)$, where

$$
\begin{aligned}
& f_{2}\left(\alpha_{2}\right)=\frac{-\gamma_{0}}{1+\left(1-\alpha_{2}\right) \gamma_{0}} \log _{2}\left(1+\alpha_{2} \gamma_{2}\right) \\
& f_{1}\left(\alpha_{2}\right)=\left(\frac{-2 \gamma_{0}}{\left.1+\left(1-\alpha_{2}\right) \gamma_{0}\right)}+\frac{\gamma_{2}}{1+\alpha_{2} \gamma_{2}}\right) \log _{2}\left(1+\gamma_{1}\right) \\
& f_{0}\left(\alpha_{2}\right)=\frac{-\gamma_{2}}{1+\alpha_{2} \gamma_{2}} \log _{2}\left(1+\left(1-\alpha_{2}\right) \gamma_{0}\right) .
\end{aligned}
$$

In fact, the optimal $\alpha_{2}$ cannot be written in closed form, so we proceed by approximations to determine the sign of $f\left(\alpha_{2}\right)$. When $\gamma_{0} \ll \gamma_{2}$ and $\gamma_{0} \ll 1, f\left(\alpha_{2}\right)$ can be approximated by $\frac{\gamma_{2}}{1+\alpha_{2} \gamma_{2}} \log _{2}\left(1+\gamma_{1}\right)$, so $f\left(\alpha_{2}\right) \geq 0$. Therefore, $R_{\mathrm{SC}-2 \mathrm{~W}}$ increases with $\alpha_{2}$ and is optimal for

$$
\alpha_{2}=\alpha_{2}^{*} \text {. }
$$

Concerning $\alpha_{1}$, we derive from the constraint $C\left(\alpha_{1} \gamma_{1}\right)<$ $C\left(\alpha_{2} \gamma_{2}\right)$ that $\alpha_{1}<\hat{\alpha}_{1}$, where $\hat{\alpha}_{1}$ is defined as

$$
\hat{\alpha}_{1}=\frac{\gamma_{2}-\gamma_{0}}{\gamma_{0} \gamma_{1}}
$$

For comparing $\hat{\alpha}_{1}$ and $\alpha_{1}^{*}$, we derive $\hat{\alpha}_{1}-\alpha_{1}^{*}=\frac{\gamma_{2}-\gamma_{1}}{\gamma_{0} \gamma_{1}}$, which is always positive since we assumed $\gamma_{2}>\gamma_{1}$. As $R_{\mathrm{SC}-2 \mathrm{~W}}$ is independent of $\alpha_{1}$ in this case, any value of $\alpha_{1}$ satisfying 
$\alpha_{1}^{*} \leq \alpha_{1}<\hat{\alpha}_{1}$ may be chosen; we may choose in particular

$$
\alpha_{1}=\alpha_{1}^{*} \text {. }
$$

Finally,

$$
R_{\mathrm{SC}-2 \mathrm{~W}}^{(c 2)}=\frac{\left[C\left(\gamma_{1}\right)+C\left(\left(1-\alpha_{2}^{*}\right) \gamma_{0}\right)+C\left(\alpha_{2}^{*} \gamma_{2}\right)\right] C\left(\gamma_{1}\right)}{2 C\left(\gamma_{1}\right)+C\left(\alpha_{2}^{*} \gamma_{2}\right)}
$$

(d) $0 \leq \alpha_{1} \leq \alpha_{1}^{*} \& 0 \leq \alpha_{2} \leq \alpha_{2}^{*}$ : In this case, we have $R_{\mathrm{b}_{1}}+R_{\mathrm{s}_{1}}=C\left(\left(1-\alpha_{1}\right) \gamma_{0}\right)+C\left(\alpha_{1} \gamma_{1}\right), R_{\mathrm{b}_{2}}+R_{\mathrm{s}_{2}}=C((1-$ $\left.\left.\alpha_{2}\right) \gamma_{0}\right)+C\left(\alpha_{2} \gamma_{2}\right)$. We consider the following subcases:

(d-1) If $C\left(\alpha_{1} \gamma_{1}\right) \geq C\left(\alpha_{2} \gamma_{2}\right)$ : then,

$$
R_{\mathrm{SC}-2 \mathrm{~W}}=\frac{\left[R_{\mathrm{b}_{1}}+R_{\mathrm{s}_{1}}+R_{\mathrm{b}_{2}}+R_{\mathrm{s}_{2}}\right] C\left(\gamma_{1}\right)}{2 C\left(\gamma_{1}\right)+C\left(\alpha_{1} \gamma_{1}\right)} .
$$

We first consider the behavior of $R_{\mathrm{SC}-2 \mathrm{~W}}$ in function of $\alpha_{1}$. The sign of the corresponding partial derivative is controlled by $g\left(\alpha_{1}\right)$, defined as $g\left(\alpha_{1}\right)=g_{2}\left(\alpha_{1}\right)+g_{1}\left(\alpha_{1}\right)+g_{0}\left(\alpha_{1}\right)$, where

$$
\begin{aligned}
g_{2}\left(\alpha_{1}\right)= & \frac{-\gamma_{1}}{1+\alpha_{1} \gamma_{1}} \log _{2}\left(1+\alpha_{2} \gamma_{2}\right) \\
g_{1}\left(\alpha_{1}\right)= & \frac{-\gamma_{0}}{1+\left(1-\alpha_{1}\right) \gamma_{0}} \log _{2}\left(1+\alpha_{1} \gamma_{1}\right) \\
& +\frac{2 \gamma_{0} \gamma_{1}\left(1+\frac{1}{\gamma_{0}}-\frac{1}{\gamma_{1}}-2 \alpha_{2}\right)}{\left(1+\left(1-\alpha_{1}\right) \gamma_{0}\right)\left(1+\alpha_{1} \gamma_{1}\right)} \log _{2}\left(1+\gamma_{1}\right) \\
g_{0}\left(\alpha_{1}\right)= & \frac{-\gamma_{1}}{1+\alpha_{1} \gamma_{1}}\left(\log _{2}\left(1+\left(1-\gamma_{1}\right) \gamma_{0}\right)\right. \\
& \left.+\log _{2}\left(1+\left(1-\alpha_{2}\right) \gamma_{0}\right)\right) .
\end{aligned}
$$

Again, obtaining the closed-form expression of the optimal $\alpha_{1}$ is very difficult, so we study the sign of $g\left(\alpha_{1}\right)$ for $\gamma_{0} \ll \gamma_{2}$ and $\gamma_{0} \ll 1$. We can show that $g\left(\alpha_{1}\right) \approx \frac{\gamma_{1}}{1+\alpha_{1} \gamma_{1}} \log _{2} \frac{\left(1+\gamma_{1}\right)^{2}}{1+\alpha_{2} \gamma_{2}}$. As the sign of $g\left(\alpha_{1}\right)$ depends on the term in the logarithm, we further assume two subcases.

(d-1-i) If $\left(1+\gamma_{1}\right)^{2} \geq 1+\alpha_{2} \gamma_{2}$, equivalent to $\alpha_{2} \leq \frac{\left(1+\gamma_{1}\right)^{2}-1}{\gamma_{2}}=$ $\overline{\alpha_{2}}$, then $g\left(\alpha_{1}\right) \geq 0$, so $R_{\mathrm{SC}-2 \mathrm{~W}}$ is an increasing function of $\alpha_{1}$. The maximum is attained when

$$
\alpha_{1}=\alpha_{1}^{*} \text {. }
$$

Next, $R_{\mathrm{SC}-2 \mathrm{~W}}$ in function of $\alpha_{2}$, follows the behavior of $\eta\left(\gamma_{2}\right)$, which is maximized at $\alpha_{2}=\tilde{\alpha}_{2}$, but $\alpha_{2}^{*} \leq \tilde{\alpha}_{2}$ as in case (c-1). Furthermore, the condition $C\left(\alpha_{1} \gamma_{1}\right) \geq C\left(\alpha_{2} \gamma_{2}\right)$ implies $\alpha_{2} \leq \hat{\alpha_{2}} \leq \alpha_{2}^{*}$. Thus, the optimal value of $\alpha_{2}$ is

$$
\check{\alpha_{2}}=\min \left\{\overline{\alpha_{2}}, \hat{\alpha}_{2}\right\}
$$

and finally

$$
\begin{aligned}
R_{\mathrm{SC}-2 \mathrm{~W}}^{(d 1 i)}= & \frac{\left[C\left(\left(1-\alpha_{1}^{*}\right) \gamma_{0}\right)+C\left(\alpha_{1}^{*} \gamma_{1}\right)\right] C\left(\gamma_{1}\right)}{2 C\left(\gamma_{1}\right)+C\left(\alpha_{1}^{*} \gamma_{1}\right)} \\
& +\frac{\left[C\left(\left(1-\check{\alpha_{2}}\right) \gamma_{0}\right)+C\left(\check{\alpha_{2}} \gamma_{2}\right)\right] C\left(\gamma_{1}\right)}{2 C\left(\gamma_{1}\right)+C\left(\alpha_{1}^{*} \gamma_{1}\right)} .
\end{aligned}
$$

(d-1-ii) If $\left(1+\gamma_{1}\right)^{2}<1+\alpha_{2} \gamma_{2}$, equivalent to $\alpha_{2}>\overline{\alpha_{2}}$, we have $g\left(\alpha_{1}\right)<0$ so $R_{\mathrm{SC}-2 \mathrm{~W}}$ is a decreasing function of $\alpha_{1}$ and the optimal value is

$$
\alpha_{1}=0 \text {. }
$$

However, the condition $C\left(\alpha_{1} \gamma_{1}\right) \geq C\left(\alpha_{2} \gamma_{2}\right)$ implies that $\alpha_{2}=0$, which is in contradiction with condition $\alpha_{2}>\overline{\alpha_{2}}$ since $\overline{\alpha_{2}}>0$. As it is not possible to satisfy these conditions simultaneously, this case is irrelevant.

(d-2) If $C\left(\alpha_{1} \gamma_{1}\right)<C\left(\alpha_{2} \gamma_{2}\right)$ : in this case, we get

$$
R_{\mathrm{SC}-2 \mathrm{~W}}=\frac{\left[R_{\mathrm{b}_{1}}+R_{\mathrm{s}_{1}}+R_{\mathrm{b}_{2}}+R_{\mathrm{s}_{2}}\right] C\left(\gamma_{1}\right)}{2 C\left(\gamma_{1}\right)+C\left(\alpha_{2} \gamma_{2}\right)} .
$$

First, to study the behavior of $R_{\mathrm{SC}-2 \mathrm{~W}}$ in function of $\alpha_{2}$, we derive the corresponding partial derivative whose sign is determined by a function $l\left(\alpha_{2}\right)=l_{2}\left(\alpha_{2}\right)+l_{1}\left(\alpha_{2}\right)+l_{0}\left(\alpha_{2}\right)$, where

$$
\begin{aligned}
l_{2}\left(\alpha_{2}\right)= & \frac{-\gamma_{0}}{1+\left(1-\alpha_{2}\right) \gamma_{0}} \log _{2}\left(1+\alpha_{2} \gamma_{2}\right) \\
l_{1}\left(\alpha_{2}\right)= & \left(\frac{-2 \gamma_{0}}{\left.1+\left(1-\alpha_{2}\right) \gamma_{0}\right)}+\frac{2 \gamma_{2}}{1+\alpha_{2} \gamma_{2}}\right) \\
& . \log _{2}\left(1+\gamma_{1}\right)-\frac{\gamma_{2}}{1+\alpha_{2} \gamma_{2}} \log _{2}\left(1+\alpha_{1} \gamma_{1}\right) \\
l_{0}\left(\alpha_{2}\right)= & \frac{-\gamma_{2}}{1+\alpha_{2} \gamma_{2}}\left(\log _{2}\left(1+\left(1-\alpha_{1}\right) \gamma_{0}\right)\right. \\
& \left.+\log _{2}\left(1+\left(1-\alpha_{2}\right) \gamma_{0}\right)\right) .
\end{aligned}
$$

Assuming $\gamma_{0} \ll \gamma_{2}$ and $\gamma_{0} \ll 1$, we obtain the approximation $l\left(\alpha_{2}\right) \approx \frac{\gamma_{2}}{1+\alpha_{2} \gamma_{2}} \log _{2}\left(\frac{\left(1+\gamma_{1}\right)^{2}}{1+\alpha_{1} \gamma_{1}}\right)$. Since $0<\alpha_{1} \gamma_{1}<\gamma_{1}$, we have always $l\left(\alpha_{2}\right)>0$ and thus $R_{\mathrm{SC}-2 \mathrm{~W}}$ increases with $\alpha_{2}$, so the maximum is attained for

$$
\alpha_{2}=\alpha_{2}^{*} \text {. }
$$

Concerning $\alpha_{1}$, with the same derivations as in case (b), we can conclude that the maximum is achieved for

$$
\alpha_{1}=\alpha_{1}^{*} \text {. }
$$

Finally, we obtain

$$
\begin{aligned}
R_{\mathrm{SC}-2 \mathrm{~W}}^{(d 2)}= & \frac{\left[C\left(\left(1-\alpha_{1}^{*}\right) \gamma_{0}\right)+C\left(\alpha_{1}^{*} \gamma_{1}\right)\right] C\left(\gamma_{1}\right)}{2 C\left(\gamma_{1}\right)+C\left(\alpha_{2}^{*} \gamma_{2}\right)} \\
& +\frac{\left[C\left(\left(1-\alpha_{2}^{*}\right) \gamma_{0}\right)+C\left(\alpha_{2}^{*} \gamma_{2}\right)\right] C\left(\gamma_{1}\right)}{2 C\left(\gamma_{1}\right)+C\left(\alpha_{2}^{*} \gamma_{2}\right)} .
\end{aligned}
$$

The whole analysis revealed that in most cases, $\alpha_{1}=\alpha_{1}^{*}$ and $\alpha_{2}=\alpha_{2}^{*}, \hat{\alpha}_{2}$ are optimal values. Thus, the best pair among $\left(\alpha_{1}^{*}, \alpha_{2}^{*}\right)$ and $\left(\alpha_{1}^{*}, \hat{\alpha}_{2}\right)$ will be used in our $S C-2 W$ relaying scheme, which greatly simplifies the power allocation procedure since there is no need to cover all the different cases. If $\gamma_{0}<\frac{\gamma_{j}}{1+\gamma_{j}}$, e.g., $\alpha_{j}^{*}>1$, we set $\alpha_{j}=1$, which means that we use multi-hop transmission on the $A_{j}-A_{k}$ link.

\section{Bounding Reference Schemes for Half-Duplex Two- Way Channel}

In the previous section, we have seen that in most cases, 
a near-optimal achievable rate was attained when $\alpha_{1}=\alpha_{1}^{*}$ and $\alpha_{2}=\alpha_{2}^{*}$ (or $\alpha_{2}=\hat{\alpha}_{2}$ ). That is, the closed-form expression of the optimal parameters was intractable in some cases. Therefore, we compare the performance of the proposed scheme with the achievable sum rate/capacity of two references schemes studied in [20], namely the 3-step inner bound referred as $T D B C$ bound and the 2-step capacity referred as $M A B C$ bound, both under the half-duplex constraint. $T D B C$ takes into account any side information that a node may acquire when it is not transmitting. Under the fairness constraint that both users transmit over the same time ratio $\delta$, the achievable sum rate of the $T D B C$ bound is

$$
\begin{aligned}
R_{\mathrm{TDBC}}= & \min \left(\delta C\left(\gamma_{1}\right), \delta C\left(\gamma_{0}\right)+(1-2 \delta) C\left(\gamma_{2}\right)\right) \\
& +\min \left(\delta C\left(\gamma_{2}\right), \delta C\left(\gamma_{0}\right)+(1-2 \delta) C\left(\gamma_{1}\right)\right)
\end{aligned}
$$

As in [20], we use the optimal $\delta$ satisfying $0<\delta<1$ and $1-2 \delta>0$. For the $M A B C$ bound, under the same fairness constraint, we can write its sum rate capacity as

$$
R_{\mathrm{MABC}}=\min \left(R_{\mathrm{MABC}}^{1}, \delta C\left(\gamma_{1}+\gamma_{2}\right)\right) .
$$

with $0<\delta<1$ and where we define

$$
\begin{aligned}
R_{\mathrm{MABC}}^{1}= & \min \left(\delta C\left(\gamma_{1}\right),(1-\delta) C\left(\gamma_{2}\right)\right) \\
& +\min \left(\delta C\left(\gamma_{2}\right),(1-\delta) C\left(\gamma_{1}\right)\right) .
\end{aligned}
$$

\section{Existing Reference Schemes}

The following benchmark schemes are considered for comparison: direct transmission where the relay is not used, multi-hop transmission where the direct link is ignored, a direct extension to the two-way relaying case of the SC scheme proposed in [5], and finally the conventional DF-NC scheme which uses three steps [10].

Direct transmission: Simply, $A_{1}$ sends $M R_{\mathrm{b}_{1}}$ bits during step 1 and $A_{2}$ sends $M R_{\mathrm{b}_{2}}$ bits during step 2, giving a rate

$$
R_{\mathrm{dir}}=\frac{R_{\mathrm{b}_{1}}+R_{\mathrm{b}_{2}}}{2}=C\left(\gamma_{0}\right)
$$

given the assumption of channel reciprocity.

Multi-hop transmission: In this case, 4 steps are required: $A_{1}$ transmits $M R_{1}$ bits in Step 1, then $A_{\mathrm{R}}$ forwards them to $A_{2}$ in Step 2, then $A_{2}$ transmits $M R_{2}$ bits in Step 3, which are forwarded to $A_{1}$ in Step 4 . Thus,

$$
R_{\mathrm{mh}}=\frac{R_{1}+R_{2}}{\left(1+\frac{R_{1}}{R_{\mathrm{r}_{2}}}\right)+\left(1+\frac{R_{2}}{R_{\mathrm{r}_{1}}}\right)}=\frac{C\left(\gamma_{1}\right) C\left(\gamma_{2}\right)}{C\left(\gamma_{1}\right)+C\left(\gamma_{2}\right)} .
$$

SC transmission: the scheme in [5] is applied for both directions: $A_{1}$ transmits $M\left(R_{\mathrm{b}_{1}}+R_{\mathrm{s}_{1}}\right)$ bits to $A_{\mathrm{R}}$ which forwards $M R_{\mathrm{s}_{1}}$ bits to $A_{2}$. Then $A_{2}$ transmits $M\left(R_{\mathrm{b}_{2}}+R_{\mathrm{s}_{2}}\right)$ bits to $A_{\mathrm{R}}$ which forwards $M R_{\mathrm{s}_{2}}$ bits to $A_{1}$. Finally, $A_{2}$ recovers the remaining $M R_{\mathrm{b}_{1}}$ bits by canceling the contribution of the $M R_{\mathrm{s}_{1}}$ bits from the signal received in Step 1, and likewise for $A_{1}$. Thus, the achievable rate is written

$$
\begin{aligned}
R_{\mathrm{sc}}= & \frac{R_{\mathrm{b}_{1}}+R_{\mathrm{s}_{1}}+R_{\mathrm{b}_{2}}+R_{\mathrm{s}_{2}}}{1+\frac{R_{\mathrm{s}_{1}}}{R_{\mathrm{r}_{2}}}+1+\frac{R_{\mathrm{s}_{2}}}{R_{\mathrm{r}_{1}}}} \\
= & \frac{2 C\left(\gamma_{1}\right) C\left(\gamma_{2}\right)}{C\left(\gamma_{1}\right)+C\left(\gamma_{2}\right)+\log _{2}\left(\frac{\gamma_{1} \gamma_{2}}{\gamma_{0}^{2}}\right)}
\end{aligned}
$$

DF-NC transmission: This refers to the conventional DFNC scheme as in [10], which counts three steps but where the direct link is ignored: first, $A_{1}$ transmits $M R_{1}$ bits to $A_{\mathrm{R}}$, then $A_{2}$ transmits $M R_{2}$ bits to $A_{\mathrm{R}}$. Finally, $A_{\mathrm{R}}$ sends the XORed packet which length is equal to the higher number of bits among $M R_{1}$ and $M R_{2}$. Moreover, the relay transmission is adapted to the lowest link among $\gamma_{1}$ and $\gamma_{2}$. Thus, the achievable rate for this three step scheme is written

$$
R_{\mathrm{dfnc}}=\frac{R_{1}+R_{2}}{2+\frac{\max \left(R_{1}, R_{2}\right)}{\min \left(R_{\mathrm{r} 1}, R_{\mathrm{r} 2}\right)}}=\frac{C\left(\gamma_{1}\right)\left[C\left(\gamma_{1}\right)+C\left(\gamma_{2}\right)\right]}{2 C\left(\gamma_{1}\right)+C\left(\gamma_{2}\right)} .
$$

\section{Numerical Results}

The analytical results obtained previously are verified numerically. Figure 3 shows the contour graph of $R_{\mathrm{SC}-2 \mathrm{~W}}$ for $\alpha_{1}$ and $\alpha_{2}$ varying in $[0,1]$, where the two relay paths have the same SNRs with $\gamma_{0}=5 \mathrm{~dB}, \gamma_{1}=20 \mathrm{~dB}$ and $\gamma_{2}=20 \mathrm{~dB}$. Here, we have $\alpha_{1}^{*}=0.31$ and $\alpha_{2}^{*}=0.31$. We can see that the maximum rate is obtained for $\left(\alpha_{1}, \alpha_{2}\right)=\left(\alpha_{1}^{*}, \alpha_{2}^{*}\right)$. Next, Fig. 4 shows the case where the two relayed paths have a higher SNR than the direct path: $\gamma_{0}=5 \mathrm{~dB}, \gamma_{1}=15 \mathrm{~dB}$ and $\gamma_{2}=20 \mathrm{~dB}$. In this case, we have $\alpha_{1}^{*}=0.28$ and $\alpha_{2}^{*}=0.31$. Figure 4 shows that the maximum rate is attained at $\alpha_{1}=\alpha_{1}^{*}$ but at a different value of $\alpha_{2}$ than $\alpha_{2}^{*}$. Since we do not have $\gamma_{0} \ll 1$, we cannot guarantee that the optimum is attained at $\alpha_{2}^{*}$ as suggested by the approximations in cases (c-2) or (d-2) in Sect. 4. But as the potential curves show, the value of $R_{\mathrm{SC}-2 \mathrm{~W}}$ attained at $\left(\alpha_{1}^{*}, \alpha_{2}^{*}\right)$ is extremely close to optimal.

We now compare the proposed scheme with the bounding and reference schemes defined in Sects. 5 and 6. The achievable rates of all the schemes are plotted in Fig. 5 for different values of $\gamma_{0}$ and where $\gamma_{1}=\gamma_{2}=20 \mathrm{~dB}$. We can

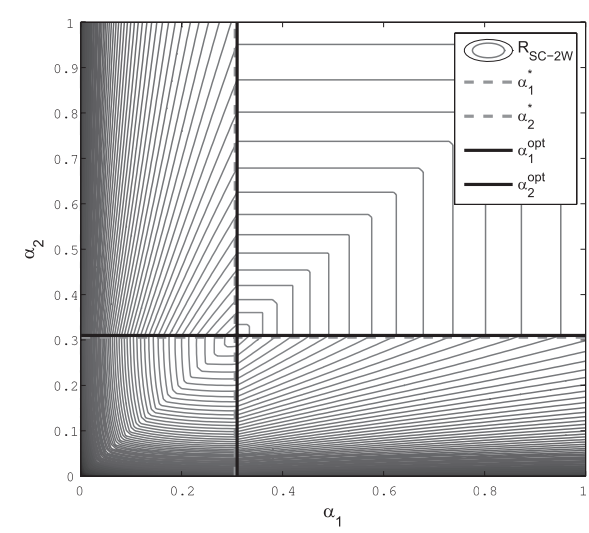

Fig. 3 Contour of the achievable rate of the proposed SC two-way relaying scheme, $\alpha_{1}^{*}=\alpha_{1}^{o p t}$ and $\alpha_{2}^{*}=\alpha_{2}^{o p t} ; \gamma_{0}=5 \mathrm{~dB}, \gamma_{1}=20 \mathrm{~dB}, \gamma_{2}=20 \mathrm{~dB}$. 


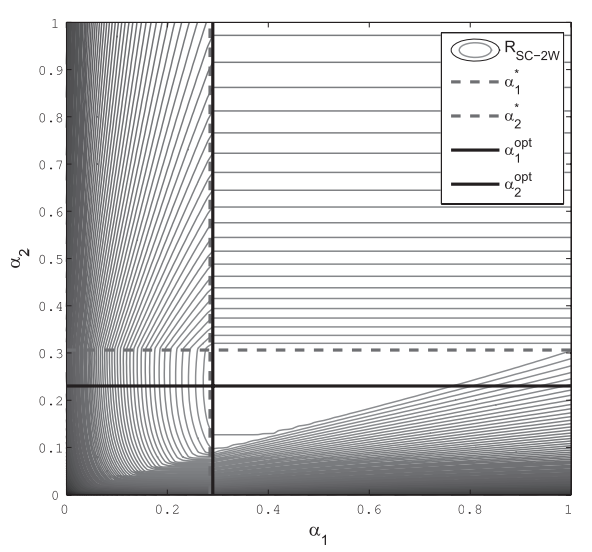

Fig. 4 Contour of the achievable rate of the proposed SC two-way relaying scheme, $\alpha_{1}^{*}=\alpha_{1}^{o p t}$ and $\alpha_{2}^{*} \neq \alpha_{2}^{o p t} ; \gamma_{0}=5 \mathrm{~dB}, \gamma_{1}=15 \mathrm{~dB}, \gamma_{2}=20 \mathrm{~dB}$.

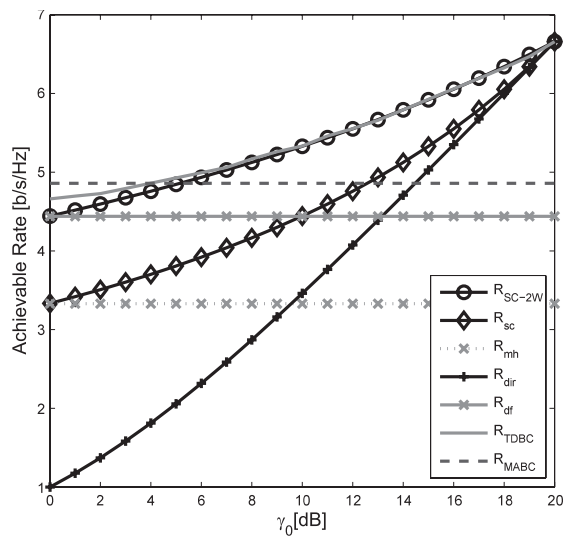

Fig. 5 Achievable rate for the proposed two-way SC relaying scheme and reference schemes, with $\gamma_{1}=\gamma_{2}=20 \mathrm{~dB}$.

see that, compared to the existing schemes, our proposed scheme achieves the best performance, while closely approaching the 3-step TDBC bound. Our scheme even outperforms the sum rate capacity of the 2-step $M A B C$ bound for $\gamma_{0} \geq 5 \mathrm{~dB}$. Thus, over a large range of SNRs, our 3step suboptimal scheme is able to achieve a larger sum rate than the optimal half-duplex 2 -step scheme, by efficient utilization of the direct transmissions. Moreover, significant gain is obtained compared to the direct extension to the two-way scenario of the SC scheme in [5], and also compared to the conventional DF-NC scheme. Note that when $\gamma_{0}=\gamma_{1}=\gamma_{2}=20 \mathrm{~dB}$, all the schemes based on SC converge to the direct transmission scheme, since $\alpha_{1}=\alpha_{2}=0$ at that point.

In Fig. 6, we show the performance of the same schemes but assuming $\gamma_{1}=17 \mathrm{~dB}$ and $\gamma_{2}=20 \mathrm{~dB}$. Again, the proposed scheme achieves the best sum rate among existing schemes, while approaching the 3-step $T D B C$ bound and outperforming the 2-step $M A B C$ bound over a large SNR region. For $\gamma_{0}>\gamma_{1}$, we can see that all the schemes that make use of the direct link achieve the same rate, i.e., it becomes optimal to use the direct path only since the direct link has a higher SNR than the relayed link $A_{1}-A_{\mathrm{R}}$.

Finally, we evaluate our proposed scheme for differ-

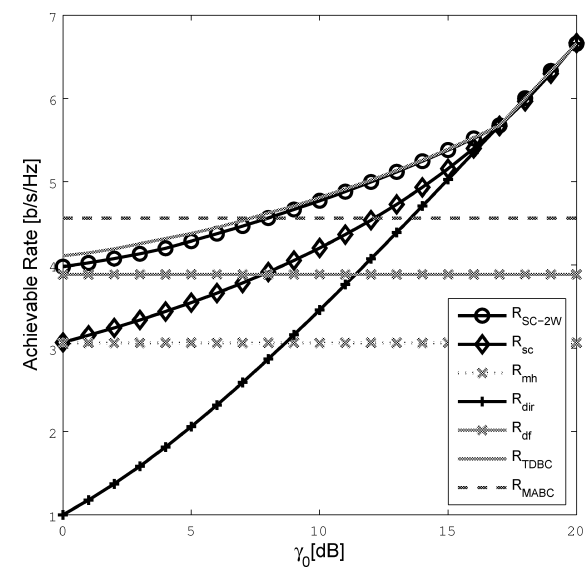

Fig. 6 Achievable rate for the proposed two-way SC relaying scheme and reference schemes, with $\gamma_{1}=17 \mathrm{~dB}, \gamma_{2}=20 \mathrm{~dB}$.

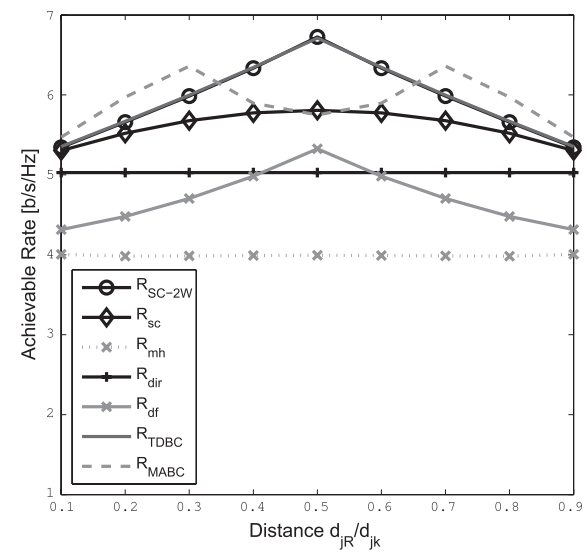

Fig. 7 Achievable rate for the proposed two-way SC relaying scheme and reference schemes, for different $A_{j}-A_{\mathrm{R}}$ distances, $\gamma_{0}=15 \mathrm{~dB}$.

ent $A_{j}-A_{\mathrm{R}}$ distances, where $A_{j}, A_{\mathrm{R}}$ and $A_{k}$ are assumed to be aligned, with $j, k \in\{1,2\}, j \neq k . d_{j \mathrm{R}}$ denotes the $A_{j-}$ $A_{\mathrm{R}}$ distance and $d_{j k}$, the $A_{j}-A_{k}$ distance. We use the definitions in [5], where for a given $\gamma_{0}$ and a given ratio $d_{j \mathrm{R}} / d_{j k}$, SNRs $\gamma_{1}$ and $\gamma_{2}$ are determined by $\gamma_{1}=\gamma_{0}\left(d_{j k} / d_{j \mathrm{R}}\right)^{v}$ and $\gamma_{2}=\gamma_{0}\left(d_{j k} /\left(d_{j k}-d_{j \mathrm{R}}\right)\right)^{v}$, where $v$ is the path loss coefficient. This ensures that $\gamma_{1}>\gamma_{0}$ and $\gamma_{2}>\gamma_{0}$. Curves are plotted in Fig. 7 for $\gamma_{0}=10 \mathrm{~dB}$. They show that for all distance ratios, our proposed scheme outperforms the other existing schemes, while approaching tightly the 3-step TDBC bound. Our scheme also outperforms the 2-step $M A B C$ bound as the relay approaches the middle distance between the 2 nodes. The largest gain of the proposed two-way SC relaying scheme against the existing schemes and $M A B C$ occurs when the relay is placed at half distance, i.e., when the two relayed links $A_{j}-A_{\mathrm{R}}$ and $A_{\mathrm{R}}-A_{k}$ are equal, as the amount of time required for the third step is minimized.

\section{Conclusion}

In this work, we have considered the problem of two-way relaying based on half-duplex DF transmission, and have 
proposed a new SC based NC scheme, namely the SC twoway relaying scheme. The key points of our scheme are that it takes advantage of the direct link, allowing its specific design into three steps, and that it provides a practical and efficient power allocation among the superposed layers, derived by analysis. The proposed power division coefficients are practical in a sense that each node only needs the knowledge of its own direct and relayed links. The simulation results have shown that the proposed scheme outperformed the other existing or reference schemes, while performing very closely to the performance upper-bound.

Superposition Coding and Network Coding are promising techniques to enhance the performance of relay systems. In the future work, we plan to investigate different scenarios that may benefit from the SC-based approach, such as a system where multiple nodes are supported by several relays.

\section{Acknowledgment}

This work was supported by the Grant-in-Aid for JSPS Fellow no. 204205 from the Ministry of Education, Science, Sports, and Culture of Japan.

\section{References}

[1] R. Pabst, et al., "Relay-based deployment concepts for wireless and mobile broadband radio," IEEE Wireless Commnu. Mag., vol.42, no.9, pp.80-89, Sept. 2004.

[2] J.N. Laneman, D.N.C. Tse, and G.W. Wornell, "Cooperative diversity in wireless networks: Efficient protocols and outage behavior," IEEE Trans. Inf. Theory, vol.50, no.12, pp.3062-3080, Dec. 2004.

[3] Y. Zhao, R. Adve, and T.J. Lim, "Improving amplify-and-forward relay networks: Optimal power allocation versus selection," Proc. IEEE International Symposium On Information Theory, Seattle, WA, July 2006.

[4] M. Kaneko, K. Hayashi, P. Popovski, K. Ikeda, H. Sakai, and R. Prasad, "Amplify-and-forward cooperative diversity schemes for multi-carrier systems," IEEE Trans. Wirel. Commun., vol.7, no.5, pp.1845-1850, May 2008.

[5] P. Popovski and E. de Carvalho, "Improving the rates in wireless relay systems through superposition coding," IEEE Trans. Wireless Commun., vol.7, no.12, pp.4831-4836, Dec. 2008.

[6] T. Cover, "Broadcast channels," IEEE Trans. Inf. Theory, vol.18, no.1, pp.2-14, Jan. 1972

[7] T.M. Cover and J.A. Thomas, Elements of Information Theory, John Wiley \& Sons, 2006

[8] L. Ping, J. Tong, X. Yuan, and Q. Guo, "Superposition coded modulation and iterative linear MMSE detection," IEEE J. Sel. Areas Commun., vol.27, no.6, pp.995-1004, Aug. 2009.

[9] S. Katti, D. Katabi, W. Hu, H. Rahul, and M. Medard, "The importance of being opportunistic: Practical network coding for wireless environments," A. Allerton Conf. Commun., Control and Comput., Sept. 2005.

[10] P. Popovski and H. Yomo, "Bi-directional amplification of throughput in a wireless multi-hop network," IEEE VTC-Spring, pp.588593, Melbourne, Australia, May 2006

[11] P. Popovski and H. Yomo, "The anti-packets can increase the achievable throughput of a wireless multi-hop network," IEEE ICC, pp.3885-3890, Istanbul, Turkey, June 2006.

[12] P. Larsson, N. Johansson, and K.-E. Sunell, "Coded Bi-directional Relaying," IEEE VTC-Spring, pp.851-855, Melbourne, Australia May 2006.

[13] S. Katti, H. Rahul, W. Hu, D. Katabi, M. Medard, and J. Crowcoft,
"Xors in the air: Practical wireless network coding," ACM Sigcomm, Pisa, Italy, Sept. 2006.

[14] P. Popovski and H. Yomo, "Physical network coding in two-way wireless relay channels," IEEE ICC, pp.707-712, Glasgow, Scotland, June 2007.

[15] R. Ahlswede, N. Cai, S.-Y.R. Li, and R.W. Yeung, "Network information flow," IEEE Trans. Inf. Theory, vol.46, no.4, pp.1204-1216, July 2000.

[16] The Network Coding Homepage. [Online] www.networkcoding. info.

[17] L. Xiao, T.E. Fuja, J. Kliewer, and D.J. Costello, "A network coding approach to cooperative diversity," IEEE Trans. Inf. Theory, vol.53, no.10, pp.3714-3722, Oct. 2007.

[18] Z. Ding, K.K. Leung, D.L. Goeckel, and D. Towsley, "On the study of network coding with diversity," IEEE Trans. Wirel. Commun., vol.8, no.3, pp.1247-1259, March 2009.

[19] S. Fu, K. Lu, Y. Qian, and M. Varanasi, "Cooperative network coding for wireless ad-hoc networks," IEEE Globecom, Washington, DC, Nov. 2007.

[20] S.J. Kim, P. Mitran, and V. Tarokh, "Performance bounds for bidirectional coded cooperation protocols," IEEE ICDCSW, Toronto, Canada, June 2007.

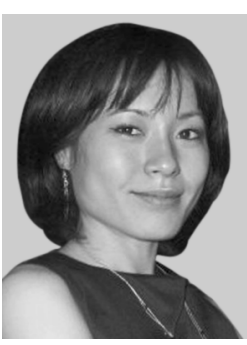

Megumi Kaneko received her B.S. and MSc. degrees in communication engineering in 2003 and 2004 from Institut National des Télécommunications (INT), France, jointly with a MSc. from Aalborg University, Denmark, where she received her Ph.D. degree in 2007. From January to July 2007, she was a visiting researcher in Kyoto University, Kyoto, Japan, and a JSPS post-doctoral fellow from April 2008 to August 2010. She is currently an Assistant Professor in the Department of Systems Science, Graduate School of Informatics, Kyoto University. Her research interests include wireless communication, protocol design and communication theory. She received the 2009 Ericsson Young Scientist Award and the IEEE Globecom'09 Best Paper Award in Wireless Communications Symposium.

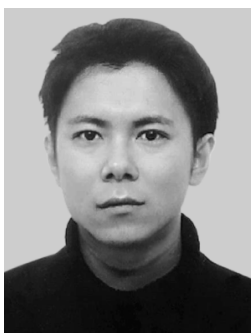

Kazunori Hayashi received the B.E., M.E and $\mathrm{Ph} . \mathrm{D}$. degrees in communication engineering from Osaka University, Osaka, Japan, in 1997, 1999 and 2002, respectively. Since 2002, he has been with the Department of Systems Science, Graduate School of Informatics, Kyoto University. He is currently an Associate Professor there. His research interests include digital signal processing for communication systems.

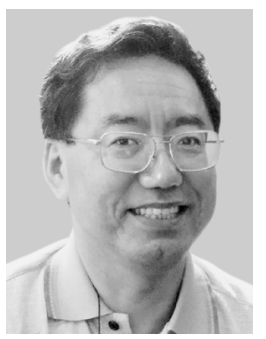

Hideaki Sakai received the B.E. and D.E. degrees in applied mathematics and physics from Kyoto University, Kyoto, Japan, in 1972 and 1981, respectively. From 1975 to 1978 , he was with Tokushima University. He is currently a Professor in the Department of Systems Science, Graduate School of Informatics, Kyoto University. He spent 6 months from 1987 to 1988 at Stanford University as a Visiting Scholar. His research interests are in the areas of adaptive and statistical signal processing. He served as an associate editor of IEEE Trans. Signal Processing from Jan. 1999 to Jan. 2001. He is a Fellow of IEEE. 